\title{
Enteroparásitos con potencial Zoonótico en Pacientes Pediátricos del Hospital de Andahuaylas - Apurímac
}

\author{
Parasites with zoonotic potential founded in children in Apurimac, Perú. \\ Faride Altamirano${ }^{1}$, Ricardo López², Nidia Puray ${ }^{1}$
}

\section{RESUMEN}

Objetivo: Determinar la prevalencia de enteropárasitos con potencial zoonótico en pacientes pediátricos que fueron atendidos en el Hospital de Andahuaylas durante los años 2008 a 2010. Metodología: A partir de 460 historias clínicas de pacientes pediátricos se recogieron datos de la edad, sexo, procedencia y resultados del examen coproparasitológico. Resultados y conclusiones: Se encontró que el 47,6\% (219/460) de niños tenía algún parásito con potencial zoonótico. Los porcentajes individuales de los parásitos con potencial zoonótico hallados fueron: Giardia intestinalis 25,9\% (119/460), Entamoeba coli 18,5\% (85/460), Blastocystis sp. 14,4\% (66/460), Hymenolepis nana 2,6\% (12/460), Entamoeba hystolitica 0,4\% (2/460), Taenia sp. 0,4\% (2/460) y Strongyloides sp. $0,2 \%$ (1/460). Se muestran adicionales análisis porcentuales.

PALABRAS CLAVE: Hospital de Andahuaylas, niños, zoonosis parasitaria.

\begin{abstract}
Aim: To determine the prevalence of intestinal parasites with zoonotic potential in pediatric patients who attend the Andahuaylas Hospital during 2008 to 2010. Methodology: By using 460 pediatric patients medical records specific data of sex, origin and coproparasitological examination report were collected. Results and conclusions: We found $47,6 \%(219 / 460)$ of children with parasites with zoonotic potential. Giardia intestinalis had the highest prevalence with 25,9\% (119/460), Entamoeba coli 18,5\% (85/460), Blastocystis sp. 14,4\% (66/460), Hymenolepis nana 2,6\% (12/460), Entamoeba hystolitica 0,4\% (2/460), Taenia sp. 0,4\% (2/460) and Strongyloides sp. 0,2\%. We show further percental analysis.
\end{abstract}

KEY WORDS: Andahuaylas hospital, children, parasitic zoonoses.

\section{INTRODUCCIÓN}

Entre los enteropárasitos con potencial zoonótico se encuentra Giardia sp. que es un parásito cosmopolita de los humanos, perros y gatos (Bowman et al., 2002; Murray et al., 2006). Se ha observado quistes de Entamoeba histolytica en las heces de gatos infectados con quistes de seres humanos. Los gatos no pueden infectarse con las otras especies de Entamoeba que infectan a los humanos (Bowman et al., 2002). Cryptosporidium spp. se ha encontrado en perros y gatos callejeros, actualmente estudiadas por su importancia médica como un peligroso parásito oportunista y contaminante de agua al igual que Giardia sp. (Acha y Szyfres, 2003), El Blastocystis $s p$. es un parásito común en un amplio rango de hospederos mamíferos, aves y reptiles, incluidos los animales domésticos los cuales presentan tasas de prevalencia muy variables (Llop et al., 2001; Abe et al., 2002), la prevalencia en los trabajadores en contacto con animales es más alto que en una población normal (17\%) (Yan et al., 2007; WHO, 2011). Entamoeba coli es la más común de todas las amebas en humanos y es considerada como un comensal del

\footnotetext{
${ }^{1}$ Facultad de Ciencias Agropecuarias, Universidad Alas Peruanas

${ }^{2}$ Instituto Nacional de Salud, Ministerio de Salud
} 
tracto gastrointestinal del humano (Botero y Restrepo, 1998; Llop et al., 2001; Saredi, 2002; Rojas, 2004; Murray et al., 2006), sin embargo estudios realizados muestran prevalencias en aves silvestres, en primates en cautiverio (Figueiroa et al., 2007; Assunçao et al., 2010), y en perros (Hee, 2011; Campos et al., 2008). Hymenolepis nana es universal en el ser humano y es también un parásito habitual del ratón y la rata. Taenia $s p$. sus estadíos larvarios conocidos como cisticercos representa un riesgo significativo para la salud humana (Saredi, 2002; Murray et al., 2006). El Strongyloides $s p$. tiene una prevalencia baja, pero una distribución geográfica muy amplia, además de los humanos, los gatos, perros y primates se pueden infectar de forma natural (Bowman et al., 2004; Llanos et al., 2010).

En el Perú, se reportan altas prevalencias de parasitismo intestinal en niños (Marcos et al., 2002; Pajuelo et al., 2005; Iannacone et al., 2006; Natividad et al., 2007; Iannacone et al., 2007; Alcaraz, 2009), dentro de los cuales, los parásitos con potencial zoonótico son reportados con más frecuencia.

El objetivo del presente estudio fue determinar el porcentaje de enteroparásitos zoonóticos diagnosticados en pacientes pediátricos del hospital de Andahuaylas.

\section{MATERIALES Y MÉTODOS}

El estudio se realizó en el Hospital Nivel II-2 Hugo Pesce Pescetto de Andahuaylas en la región Apurímac. La población objetivo fueron las historias clínicas con resultados de estudio coproparasitológico de pacientes de 0 a 15 años, de ambos géneros, atendidos en el Hospital durante el periodo 2008 a $2010(\mathrm{~N}=5010)$. El tamaño muestral se determinó mediante la fórmula de comprobación de una proporción (prevalencia referencial del 50\%, nivel de confianza del 95\% y error máximo admisible del 5\%), calculándose una muestra mínima de 384. La selección de la muestra se realizó mediante muestreo sistemático.

Las variables consideradas en el estudio fueron edad, género, procedencia (provincia) y tipos de parásitos encontrados al diagnóstico coproparasitológico. Los datos extraídos de las historias fueron registrados en

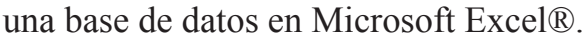

La información obtenida fue analizada mediante el programa Statistical Package for the Social Sciences (SPSS versión 19). Se realizaron pruebas de Chi-cuadrado con niveles de confianza de 95\% para determinar la asociación entre las variables demográficas y la condición parasitaria de los pacientes.

\section{RESULTADOS}

El 53,26\% (245/460) de niños estuvieron expuesto a alguna forma parasitaria intestinal. Se encontró una prevalencia de $47,6 \pm 4,67 \%$ de pacientes pediátricos con diagnóstico de enteroparásitos potencialmente zoonóticos.

Según el sexo de los niños el porcentaje de infección con parásitos con potencial zoonótico fue de $50,9 \%$ en niñas y el $44,2 \%$ en niños. Los niños de 1 a

Cuadro 1.- Muestras positivas a enteroparásitos zoonóticos según variables en pacientes pediátricos del Hospital de Andahuaylas durante los años 2008-2010 ( $\mathrm{n}=460)$

\begin{tabular}{|c|c|c|c|c|}
\hline \multirow[t]{2}{*}{ Variable } & \multirow[t]{2}{*}{ Estrato } & \multirow[t]{2}{*}{ Total de muestras } & \multicolumn{2}{|c|}{$\begin{array}{l}\text { Muestras } \\
\text { positivas }\end{array}$} \\
\hline & & & Nro. & $\%$ \\
\hline \multirow{2}{*}{$\operatorname{Sexo}^{(1)}$} & Femenino & 234 & 119 & 50,9 \\
\hline & Masculino & 226 & 100 & 44,2 \\
\hline \multirow{4}{*}{ Grupo etáreo $^{(2)}$} & $<1$ año & 39 & 7 & 17,9 \\
\hline & $01-05$ & 270 & 124 & 45,9 \\
\hline & $06-10$ & 101 & 58 & 57,4 \\
\hline & $11-15$ & 50 & 30 & 60,0 \\
\hline \multirow{3}{*}{ Provincia } & Andahuaylas & 446 & 211 & 47,3 \\
\hline & Chincheros & 11 & 7 & 63,4 \\
\hline & La Convención & 3 & 1 & 33,3 \\
\hline
\end{tabular}


Cuadro 2. Prevalencia de enteroparásitos zoonóticos en pacientes pediátricos del Hospital de Andahuaylas durante los años 2008-2010 (n=460)

\begin{tabular}{lccc}
\hline \multicolumn{1}{c}{ Tipo de Parásito } & Muestras positivas & Prevalencia (95\%) & IC \\
\hline Giardia sp. & 119 & 25,87 & 4,11 \\
Entamoeba coli & 85 & 18,48 & 3,66 \\
Blastocystis sp. & 66 & 14,35 & 3,31 \\
Hymenolepis nana & 12 & 2,61 & 1,57 \\
Taenia sp. & 2 & 0,46 & 0,38 \\
Entamoeba histolytica & 2 & 0,46 & 0,38 \\
Strongyloides sp. & 1 & 0,22 & 0,21 \\
\hline
\end{tabular}

Cuadro 3. Poliparasitismo en pacientes pediátricos atendidos en el Hospital de Andahuaylas durante los años 2008-2010 (n=460)

\begin{tabular}{lll}
\hline Poliparasitismo & $\mathbf{N}^{\mathbf{0}}$ & Porcentaje \\
\hline Monoparasitismo & 161 & 35,00 \\
Biparasitismo & 70 & 15,22 \\
Triparasitismo & 12 & 2,61 \\
Tetraparasitismo & 2 & 0,43 \\
Total & 245 & 100,00 \\
\hline
\end{tabular}

5 años de edad tuvo el mayor porcentaje de infección $(45,9 \%)$. El mayor número de niños procedían de la provincia de Andahuaylas 211, con el 47,3\% de infección (Cuadro 1).

De la muestra se obtuvo una prevalencia de Giardia sp. $25,87 \% \pm 4,11$ IC, E. coli $18,48 \% \pm 3,66$ IC, Blastocystis sp. 14,35\% $\pm 3,31 \mathrm{IC}, H$. nana $2,61 \%$ $\pm 1,57$ IC, Taenia sp. $0,46 \% \pm 0,38$ IC, E. histolytica $0,46 \% \pm 0,38$ IC y $0,22 \% \pm 0,21$ IC Strongyloides $s p$. (Cuadro 2).

El 161/460 (35,0\%) de los niños diagnosticados con al menos una forma parasitaria presentó monoparasitismo, biparasitismo 70/460 (15,22\%), triparasitismo $12 / 460(2,61 \%)$ y tetraparasitismo $2 / 460(0,43 \%)$ (Cuadro 3$)$.

\section{DISCUSIÓN}

Los resultados de éste estudio permitió determinar la prevalencia de enteroparásitos en pacientes pediátricos del Hospital de Andahuaylas en el distrito de Andahuaylas - Apurímac. Actualmente no existen estudios de prevalencia por parasitismo intestinal con potencial zoonótico en poblaciones cerradas, como en el hospital de Andahuaylas, siendo este estudio el primero en ser realizado en la institución.
En lo referente al parasitismo de los 460 pacientes pediátricos que se sometieron a un examen coproparasitológico, el 53,26\% (245/460) resultaron positivos a alguna forma de parásito intestinal, de los cuales $219(47,61 \%)$ fueron diagnosticados con parásitos intestinales zoonóticos. De los estudios realizados en niños en el país muestran altos porcentajes globales de éste tipo de parásitos (Marcos et al., 2002; Pajuelo et al., 2005; Iannacone et al., 2006; Iannacone et al., 2007; Natividad et al., 2007; Alcaraz, 2009). Siendo una causa recurrente de parasitismo en niños a nivel nacional y considerando esta prevalencia hallada como moderadamente alta.

La prevalencia de Giardia sp. obtenida fue de $25,87 \%$ (119/460) (Cuadro 2), superior a la prevalencia hallada en Lima 10,9\% (Pajuelo et al., 2005), Huaral 21,5\% (Natividad et al., 2007), Amazonas 21,9\% (Natividad et al., 2007), el resultado expuesto fue menor a los hallados en Junín 37,1\% y $32,6 \%$ (Marcos et al., 2002), se afirma que es uno de los parásitos más comunes y endémicos en el país. La segunda especie parasitaria más prevalente fue $E$. coli $(18,48 \%)$, aunque es considerada como comensal no patógeno del tracto gastrointestinal del humano, adquiere gran importancia ya que conforme con lo señalado en estudios realizados en Japón (Abe et al., 2002), Brasil (Figueiroa et al., 2007; Assunçao et al., 
2010) y Tailandia (Leeloyoova et al.,2009), existe la posibilidad de su transmisión hacia humanos que se encuentran en contacto con la heces de animales que puedan ser portadores, algunos autores consideran que los registros ocasionales de E. coli en perros son probablemente el resultado del consumo de heces de humanos (Mcpherson et al., 2000). Blastocystis sp. tuvo una prevalencia de $14,35 \% \pm 3,31 \mathrm{IC}$, similar a la hallada en escolares del distrito de Surco-Lima que fue $12,5 \%$ (Iannacone et al., 2006) . Las elevadas tasas de infección por estos parásitos reflejan una frecuencia de exposición de la población alta a la contaminación con heces. El agua pareciera ser el factor principal de estas elevadas tasas, pues como se sabe la mayoría de estos protozoarios pueden ser trasmitidas por vía hídrica. Pero también hay que considerar la falta de educación que lleva a bajas condiciones de higiene además de la escasez de recursos económicos que se ha verificado existen en la comunidad (Cordero et al., 2008; Pérez et al., 2008).

La prevalencia de $H$. nana fue $2,61 \%(12 / 460)$, porcentajes similares en Lima 4,63\% (Cordero et al., 2008) 1,0\% (Iannacone et al., 2006), considerando que la principal actividad económica en la provincia de Andahuaylas, en el área rural es la agricultura, lo que atrae la presencia de roedores, siendo éstos el principal reservorio de Hymenolepis sp. para humanos; sin embargo, en un estudio realizado en San Juan de Lurigancho en Rattus rattus y $R$. norvegicus, el parásito hallado con mayor frecuencia fue $H$. diminuta (Iannacone et al 2002). Para E. histolytica se halló una prevalencia de $0,43 \%$, comparada con las prevalencias reportadas en las regiones Junín 53\%, Cusco 38,6\% y Lima 6\% (Cornejo et al., 2010), es muy inferior, conociendo que pueden actuar como reservorios los gatos y perros principalmente, los que viven en estrecho contacto con los niños, por ser mascotas más comunes. La prevalencia de Taenia $s p$. fue de $0,43 \%$ inferior a la reportada en la región Ayacucho 1,4\% (Cordero et al., 2008), superior a la hallada en Alto Marañón, Amazonas 0,2\% (Natividad et al., 2007), esto debido al control ejercido por varias instituciones para la erradicación de la cisticercosis humana. Strongyloides $s p$. tuvo una prevalencia de $0,22 \%$, el hallazgo de un geohelminto que se presenta con mayor frecuencia en las regiones de la Amazonía tanto en animales como en humanos (Natividad et al., 2007; Farah et al., 2008), este resultado indica que pudo haber ocurrido una migración desde éstas zonas.

Sobre el poliparasitismo intestinal en pacientes pediátricos (Cuadro 3), el mayor porcentaje fue el monoparasitismo (161/460) seguido del biparasitismo $15,22 \%(70 / 460)$. El poliparasitismo, hace referencia a un hospedero con diagnosticado con varias especies de parásitos. A diferencia de los artículos consultados que muestran que el poliparasitismo se presentan con mayores porcentajes (Iannacone et al., 2007; Natividad et al., 2007). La diferencia a los resultados del presenté estudio, probablemente esto se deba a la presencia de otros factores ambientales propias del medio rural dónde se realizó el estudio.

En referencia a los enteroparásitos zoonóticos por grupo etario (Cuadro 1), se observó un mayor número de casos en el grupo de 1- 5 años que representa el $56,6 \%(124 / 219)$, éstos resultados son similares a los hallados en otros trabajos que reportan mayores porcentajes de parasitosis en niños de 1 a 5 años con el 47,1\% (Pajuelo et al., 2005). Esto se debería a su mayor vulnerabilidad, asociada a malos hábitos de higiene o el estado nutricional que predispone a este grupo, en particular, a un mayor riesgo de infección por protozoos y helmintos, encontrándose asociación entre la edad y el parasitismo zoonótico más no en el caso del sexo de los niños y el estado parasitario de acuerdo con otros autores (Pajuelo et al., 2005; Iannacone et al., 2006; Natividad et al., 2007), esto sugiere que la exposición a la parasitosis es similar.

Las provincias de procedencia de los pacientes pediátricos atendidos en el Hospital de Andahuaylas, los 446 pacientes provenientes de la provincia de Andahuaylas 211 (47,3\%) resultaron positivos, seguida por la provincia de Chincheros con 7 positivos y la provincia de La Convención perteneciente a la región Cusco con 1 paciente positivo (Cuadro 1). Este resultado refleja la población que es atendida en el Hospital de Andahuaylas proviene de ésta provincia.

\section{CONCLUSIONES}

- Los datos obtenidos en el trabajo de investigación demuestran que la prevalencia de enteroparásitos zoonóticos, al $95 \%$, es equivalente al $47,60 \% \pm$ 4,67 IC (219/460).

- El parásito con mayor prevalencia fue Giardia sp. con $25,87 \% \pm 4,11$ IC , seguida de Entamoeba coli $18,48 \% \pm 3,66$ IC y Blastocystis sp. $14,35 \% \pm 3,31$ IC (66/460).

- La cuidad de Andahuaylas presentó el 71,11\% de niños con parásitos intestinales con potencial zoonótico (211/219).

- El grupo etario más vulnerable a parasitismo intestinal estuvo comprendida entre 1-5 años $(124 / 219)$.

\section{AGRADECIMIENTOS}

A las autoridades del Hospital Hugo Pesce Pescetto de 
la provincia de Andahuaylas- Apurímac y al personal del Laboratorio Clínico, que permitieron realizar el trabajo de investigación.

\section{Correspondencia}

Faride Altamirano

Correo electrónico: faride_az@hotmail.com

\section{REFERENCIAS BIBLIOGRÁFICAS}

1. Abe N, Nagoshi M, Takami K, Sawano Y, Yoshikawa H. 2002. A survey of Blastocystis sp. in livestock, pets and zoo animals in Japan. Vet Parasitol 106 (Suppl. 3): 203-212.

2. Acha P, Szyfres B. 2003. Zoonosis y enfermedades transmisibles comunes al hombre y a los animales. $3^{\mathrm{a}}$ ed. Volumen III. Parasitosis. Washington: Organización Panamericana de la Salud. 411p

3. Alcaraz M. Giardia y Giardiosis. [Internet], [20 mayo 2009]. Disponible en: www.seimc.org/ control/revi para/pdf/Giardia.pdf. Acceso el 20 mayo de 2009.

4. Assunçao W, Rocha K, Marruaz K, Pereira M, Moreira M. 2010. Ocurrencia de hepatitis virales, helmintiasis y protozoosis en primates neotropicales procedentes de cría domiciliaria: afecciones de transmisión fecaloral con potencial zoonótico. Rev Pan-Amaz Saude 1 (Suppl. 3):57-60.

5. Botero D, Restrepo M. 1998. Parasitosis Humanas. $3^{\mathrm{a}}$ ed. Medellín: Corporación para Investigaciones Biológicas. 506p

6. Bowman D, Hendrix C, Lindsay D, Barr S. 2002. Feline clinical parasitology. Iowa: Iowa State University. 469p

7. Bowman D, Lynn R, Eberhard M. 2004. Georgis: parasitología para veterinarios. $8^{\mathrm{a}}$ ed. Madrid: Elsevier; 440p

8. Campos P, Barros M; Campos O, Braga B; Cazorla M; Albuquerque R; Carvalho M. . 2008; Parasitas zoonóticos em fezes de cães em praças públicas do município de Itabuna, Bahia, Brasil. Rev Bras Parasitol Vet 17 (Suppl. 4):206-209.

9. Cordero A, Miranda E, Segovia G, Cantoral V, Huarcaya I. 2010. Prevalencia de teniosis y seroprevalencia de cisticercosis humana en Pampa Cangallo, Ayacucho, Perú 2008. Rev Peru Med Exp Salud Pública 27 (Suppl. 4): 562-68.

10. Cornejo W, Espinoza Y, Huiza A, Pilar A, Suarez R, Sevilla C, Náquira C. Prevalencia de E. histolytica y E. dispar por Microscopía y ELISA en Muestras Fecales de una Población Urbano Marginal de Lima. [Internet], [18 septiembre 2010]. Disponible en: // sisbib.unmsm.edu.pe/bvrevistas/anales/v60 n2/e histolytica.htm

11. Farah C, Tantaleán M, Gibson K, Williams M. 2008. Prevalencia de helmintos intestinales de una población de monos maquisapas silvestres Ateles belzebuth chamek en el Parque Nacional de Manu,
Perú. Neotrop. Helminthol 2 (Suppl.1): 19-26.

12. Figueiroa M, Bianque J, Dowell De Brito M, Soares A, Magalhaes V, Alves R, Evencio A. 2002. Parásitos gastrointestinales de aves silvestres en cautiverio en el estado de Pernambuco, Brasil. Parasitol Latinoam 57:50 - 54.

13. Hee J. Review of zoonotic parasites in medical and veterinary fields in the Republic of Korea. [Internet], [23 diciembre 2011]. Disponible en: www.ncbi.nlm. nih.gov/pmc/articles/PMC2769215/?tool=pubmed

14. Iannacone J, Alvariño L. 2002. Helmintofauna de Rattus rattus (Linnaeus, 1758) y Rattus norvegicus (Berkenhout, 1769) (Rodentia: Muridae) en el distrito de San Juan de Lurigancho, Lima - Perú. Rev Peru Med Exp Salud Pública 19 (Suppl. 3):136-141.

15. Iannacone J, Alvariño L. Helmintos intestinales en escolares de Chorrillos y Pachacamac, Lima, PerúBiologist. 2007: 2 (Suppl. 1):27-34.

16. Iannacone J, Benitez M, Chirinos L. 2006. Prevalencia de infección por parásitos intestinales en escolares de primaria de Santiago de Surco, Lima, Perú. Parasitol Latinoam 61: 54 - 62.

17. Ibáñez N, Jara C, Guerra A, Díaz E. 2004. Prevalencia del enteroparasitismo en escolares de comunidades nativas del Alto Marañón, Amazonas, Perú. Rev Peru Med Exp Salud Pública 21 (Suppl. 3): 126-133.

18. Leelayoova S, Siripattanapipong S, Naaglor T, Taamasri P, Mungthin M. 2009. Prevalence of intestinal parasitic infections in military personnel and military dogs, Thailand. J Med Assoc Thai 92 (Suppl. 1):53-59.

19. Llanos M, Condori M, Ibañez T, Loza M. 2010. Parasitosis entérica en caninos (Canis familiaris) en el área urbana de Coroico, Nor Yungas. Departamento de La Paz, Bolivia.J Selva Andina Res Soc 1(Suppl. 1):37-49.

20. Llop A, Valdés M, Zuazo J. 2001. Microbiología y parasitología médicas. La Habana: Editorial Ciencias Médicas. 666p

21. Macpherson C, Meslin F, Wandeler A. 2000. Dogs, zoonoses, and public health. London: CABI Publishing. 383p

22. Marcos L, Maco V, Terashima A, Samalvides F, Gotuzzo E. 2002. Prevalencia de parasitosis intestinal en niños del valle del Mantaro, Jauja, Perú, Rev Med Hered 13(Suppl. 3): 85-90.

23. Murray P, Rosenthal K, Prüfer M. 2006. Microbiología Médica. $5^{\text {a }}$ ed. Madrid, España: Editorial Elsevier. 976p

24. Natividad I, Reyes J, Trelles de Belaunde M. Viguria Y, Yabar D, Terashima A. 2007. Presencia de Strongyloides stercoralis en un estudio sobre enteroparasitosis en escolares del asentamiento humano "La Candelaria", distrito de Chancay, provincia de Huaral, departamento de Lima. Acta méd. peruana 24 (Suppl. 3): 177-180.

25. Pajuelo G, Lujan D, Paredes B. 2005. Estudio de enteroparásitos en el Hospital de Emergencias 
Pediátricas, Lima-Perú. Rev Med Hered 16 (Suppl. 3):178-183.

26. Pérez G, Rosales M, Valdez R, Vargas F, Cordova O. 2008. Detección de parásitos intestinales en agua y alimentos de Trujillo, Perú. Rev Peru Med Exp Salud Pública 25 (Suppl. 1): 144-48.

27. Rojas M. 2004. Nosoparasitosis de los rumiantes domésticos peruanos. $2^{a}$ ed. Lima: Martegraf. 83p

28. Saredi N. 2002. Manual práctico de parasitología médica. Buenos Aires: Laboratorios Andrómaco;. $112 \mathrm{p}$
29. World Health Organization. Waterborne zoonoses: identification, cause and control. [Internet], [10 diciembre 2011]. Disponible en: www.who.int/ water_sanitation_health/diseases/zoonoses/en/

30. Yan Y, Su S, Ye Ji, Lai X, Lai R, Liao H, et al. 2007. Blastocystis $s p$. subtype 5: a possible zoonotic genotype. Parasitol. Res 101: 1527 - 1532.

Recibido: 12/05/2014

Aceptado: 29/08/2014 